\title{
Synthesis and fabrication of surface-active microparticles using membrane emulsion technique and rapid conjugation of model protein via strain-promoted azide-alkyne click chemistry in physiological conditions
}

\author{
Grace Walden ${ }^{\mathrm{a}}$, Xin Liao ${ }^{\mathrm{a}}$, Graham Riley ${ }^{\mathrm{b}}$, Simon Donell ${ }^{\mathrm{c}}$, Michael J Raxworthy ${ }^{\mathrm{d}}$ and Aram Saeed ${ }^{\mathrm{a}^{*}}$

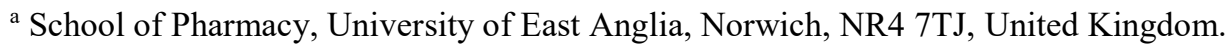 \\ ${ }^{\mathrm{b}}$ School of Biological Sciences, University of East Anglia, Norwich, NR4 7TJ, United Kingdom. \\ ${ }^{c}$ Norwich Medical School, University of East Anglia, Norwich, NR4 7TJ, United Kingdom. \\ ${ }^{\mathrm{d}}$ Neotherix Limited, York, YO1 7PR, United Kingdom.
}

\begin{abstract}
The rapid surface immobilisation of protein on monodispersed polyester microcarriers is reported. A model protein, functionalised with a dibenzocyclooctyne core, immobilises on the surface of azide-terminal polycaprolactone microcarriers within 10 minutes compared to 12 hours for other conjugation techniques and is conducted in physiological conditions and in the absence of coupling reagents.
\end{abstract}

In the field of tissue engineering and regenerative medicine, there is more demand for biomaterials that are able to provide the necessary biological cues needed to establish a natural healing environment. ${ }^{1,2}$ Therefore the design of simple and effective delivery systems for bioactive molecules, including proteins and cells, is becoming increasingly important. ${ }^{3}$ A delivery system that is able to exert spatiotemporal control over the release and presentation of biomolecules, localised to the site of action, could minimise side effects, increase therapeutic effectiveness and enhance cellular retention. ${ }^{4,5}$ An effective polymeric delivery system for proteins needs to be biodegradable at a rate comparable to the re-establishment of new tissue, and material properties. ${ }^{6}$ In addition, it needs to demonstrate little to no cytotoxicity and avoid generating an inflammatory response. ${ }^{27}$ Polymers used for delivery systems need to contain biomimetic adhesion sites for the facile conjugation of bioactive agents. ${ }^{8}$ Currently, research towards the synthesis of these types of delivery systems has been met with the challenge of choosing between natural polymers abundant with biologically active interaction sites or mechanically superior synthetic polymers lacking in useful functional groups. ${ }^{9}$

Synthetic polymers have advantageous chemical properties, such as good porosity, tuneable degradation rates and increased mechanical strength. ${ }^{10}$ These polymers, however, suffer from poor surface adhesion and an inability to easily conjugate biomolecules such as proteins or peptides that can interact with cellular components. ${ }^{9}$ For decades, polyester polymers such poly(lactic-co-glycolic acid) (PLGA), Poly(caprolactone) (PCL) and poly(lactic acid) (PLA) have been the preferred materials for delivery systems. ${ }^{19,11}$ However, these polymers are inherently difficult to work with. They lack functional groups needed to allow for easy conjugation to, or control the adhesion of, bioactive agents. ${ }^{12,13}$ This, in turn, has limited their application. ${ }^{12}$ Post-functionalisation of these polymers, in which functional groups are substituted or modified using additional reagents, has been attempted to combat this problem. ${ }^{14}$ One of the most common techniques employed to functionalise these polymers is aminolysis. ${ }^{15}$ This requires physical adsorption on the surface and the use of carbodiimide coupling reagents. ${ }^{1}$ However, as these techniques often require multi-step reactions and laborious purification processes, these delivery systems have seen little success in clinical translation. ${ }^{16}$ Recently, a new approach has been developed in which functional $\varepsilon$-caprolactone monomers can be used to synthesise polycaprolactone with a variety of functional groups. ${ }^{13}$ This is achieved by utilising the substitution of halogen groups or functionalisation of the carbonyl backbone of PCL by bromination, epoxidation or hydroxylation. ${ }^{13}$ To meet these challenges, we introduce a facile way to control the functional groups present on the polymer PCL by changing the initiator used during ring opening polymerisation (ROP). This functionalised polymer is then able to carry out the click chemistry reaction for the efficient conjugation of protein.

A heterobifunctional initiator containing a hydroxyl group at one end, and an azide group at the other can be used for the polymerisation of $\varepsilon$-caprolactone in bulk, with a catalytic amount of tin(II) 2-ethylhexanoate at $120^{\circ} \mathrm{C}$ and under an atmosphere of nitrogen. This resulted in PCL functionalised with a terminal azide (Figure 1, A). 
A) Synthesis of PCL-azide from 2-[2-(2-azidoethoxy)ethoxy]ethanol

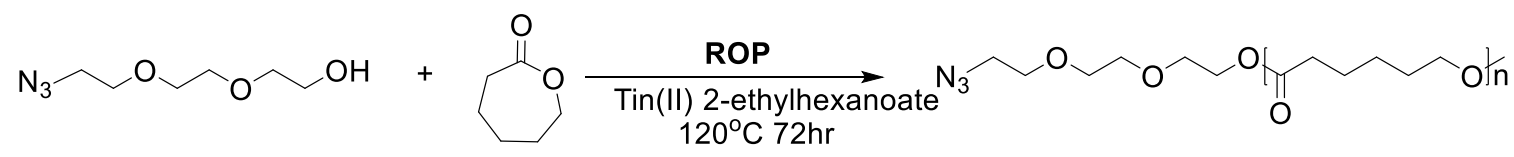

2-[2-(2-azidoethoxy)ethoxy]ethanol e-Caprolactone

PCL-azide

B) Production of PCL-azide monodispersed microparticles (membrane emulsion)

$$
\mathrm{N}_{3} \sim \mathrm{O}_{\mathrm{O}} \sim \mathrm{O}_{\mathrm{O}} \sim \mathrm{O}_{\mathrm{n}}
$$

PCL-azide dissolved in DCM

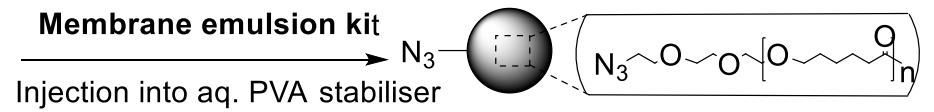

PCL-azide functionalized microparticles

C) Synthesis of Human Serum Albumin (HSA) conjugates to Dibenzocyclooctyne-maleimide

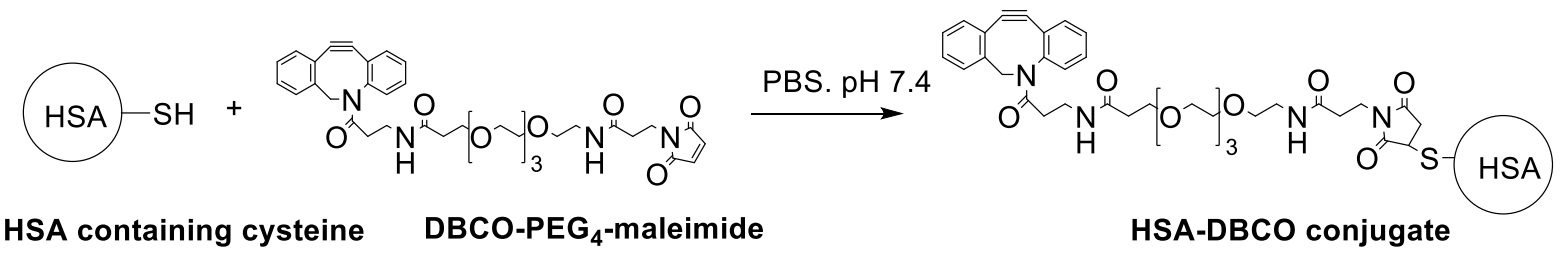

D) Immobilization of Human serum albumin conjugates to PCL-azide microparticles

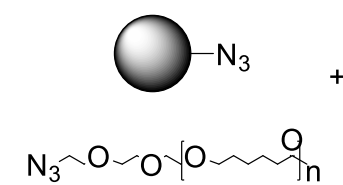

PCL-azide functionalized microparticle suspension

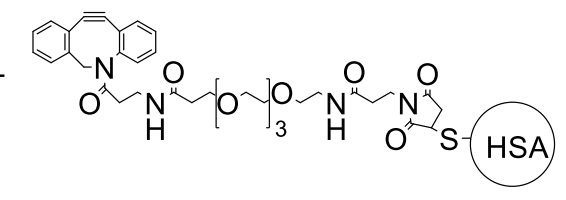

HSA-DBCO conjugate

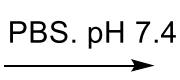

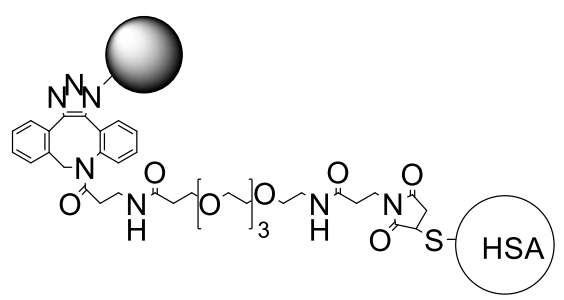

Microparticle/HSA/DBCO conjugate

Figure 1: Reaction scheme showing the synthesis of functionalised microparticles with protein immobilised on the surface. Step A shows the synthesis of a functionalised PCL-azide polymer. This is then used as the oil phase, dissolved in organic solvent, to produce microparticles via oil and water emulsion (step B). Step C shows the conjugation of protein to

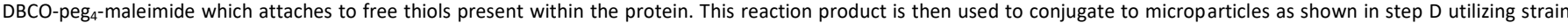
promoted azide alkyne cycloaddition.

For the ring opening polymerisation, 2[2-[2azidoethoxy)ethoxy]ethanol was used as the heterobifunctional initiator, containing a short diethylene glycol spacer to enhance its hydrophilicity. ${ }^{1 \mathrm{H}} \mathrm{NMR}$ diffusion ordered spectroscopy (DOSY) showed that the terminal azide of PCL could readily bind to a dibenzocyclooctyne (DBCO) unit within 30 minutes (see supporting information, S5). The structure and molecular weight of the purified polymer was confirmed by nuclear magnetic resonance (NMR) and gel permeation chromatography (GPC). The ${ }^{1 \mathrm{H}} \mathrm{NMR}$ showed signature peaks indicative of a polycaprolactone backbone. The number average molecular weight $(\mathrm{Mn})$ of the polymer was confirmed by GPC from samples measured in triplicate and averaged as 19502, with typical dispersity index (PDI) for ROP of 1.1, indicative of a controlled polymerisation reaction and a uniform polymer, in agreement with published work. ${ }^{17}$ Fourier transform infrared spectroscopy (FTIR) analysis of the purified PCL-azide polymer showed a peak present at $2101 \mathrm{~cm}^{-1}$, confirming the successful inclusion of the azide initiator into the backbone of the polymer (data not shown).

Next, the PCL-azide polymer was used for the fabrication of monodispersed microparticles using an oil in water membrane emulsification technique (Figure 1, B). Briefly, the membrane emulsification kit contains a glass cylinder that sits atop the injection chamber, which holds a metal membrane with uniform pores of a specified size throughout. PCL-azide polymer dissolved in dichloromethane (DCM) was used as the oil phase and injected via an automated syringe pump through the pores of the membrane into the aqueous phase containing $1 \%$ polyvinyl alcohol (PVA) as a surfactant. The PCL-azide droplets form on the surface of the membrane and are removed by a stirrer paddle 
with adjustable rotation speed. PCL-azide droplets gradually solidify, by solvent evaporation, resulting in microparticles that can be washed to remove all PVA and freeze-dried for further use. It is possible to tightly control particle size and monodispersity by altering the process parameters. The effect of increasing rotation speed and polymer concentration on particle morphology and size distribution was investigated. Polymer concentrations of 5, 10, 20, 30 and 40w/v\% PCL-azide in DCM were used and the rotation speed kept constant at 1140 RPM. It was found that as the polymer concentration increased so did the average particle size, resulting in particles of $24 \pm 0.21 \mu \mathrm{m}$ and $34 \pm 0.39 \mu \mathrm{m}$ in diameter for 5 and $40 \mathrm{w} / \mathrm{v} \%$ PCL-azide respectively (Figure 2). The span of particles represent the size distribution, and can be calculated using (D90-D10)/D50) where $\mathrm{D}$ represents the diameter which is equal to or greater than that $\%$ of particles in the sample. ${ }^{18,10}$ A size distribution of less than 1 indicates a monodisperse population of particles.
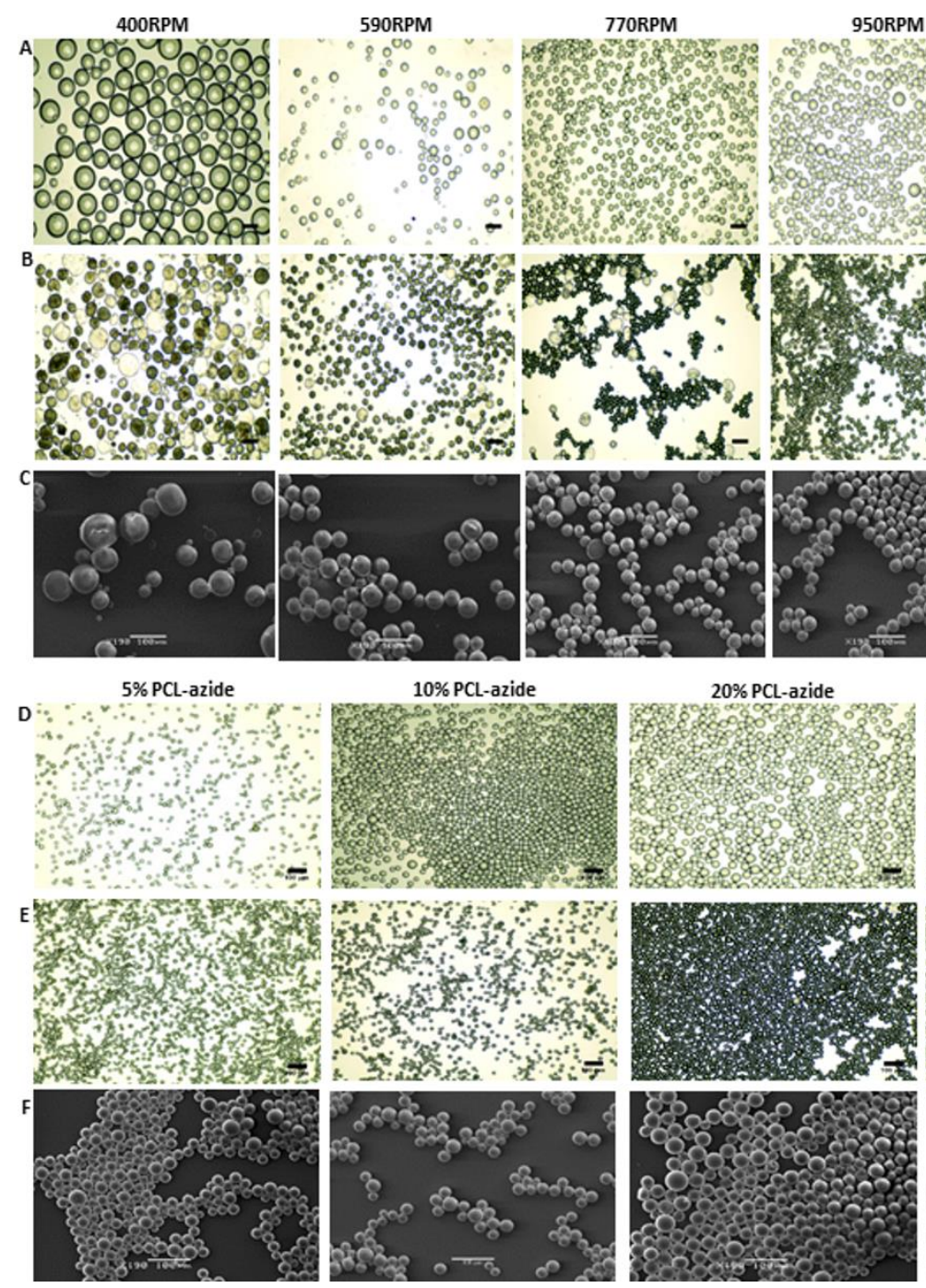

population (see supporting information, S2). The increase in particle size can be attributed to the increased viscosity, due to increased concentration of the polymer, which results in larger droplets forming on the surface of the membrane before detachment by the rotating stirrer paddle. ${ }^{19}$ Rotation speeds of 400, 590, 770, 950, 1140 and 1500 RPM were assessed, whilst polymer concentration remained constant at $10 \mathrm{w} / \mathrm{v} \%$. It was found that as rotation speed increased the average particle size decreased, resulting in particles with a diameter of $71 \pm 2.18 \mu \mathrm{m}$ and $22 \pm 0.24 \mu \mathrm{m}$ at rotation speeds of 400 and 1500 respectively. At the lowest RPM (400) it was found that, the dispersity of the microparticles was poorest, with a span of 1.2, suggesting a less monodispersed population, but this improved when the stir speed was increased (see supporting information, S2). A decrease in particle size with increased rotation speed is due to the rapid collection of particle droplets from the surface of the membrane. ${ }^{19}$ It was shown that highly monodispersed particles
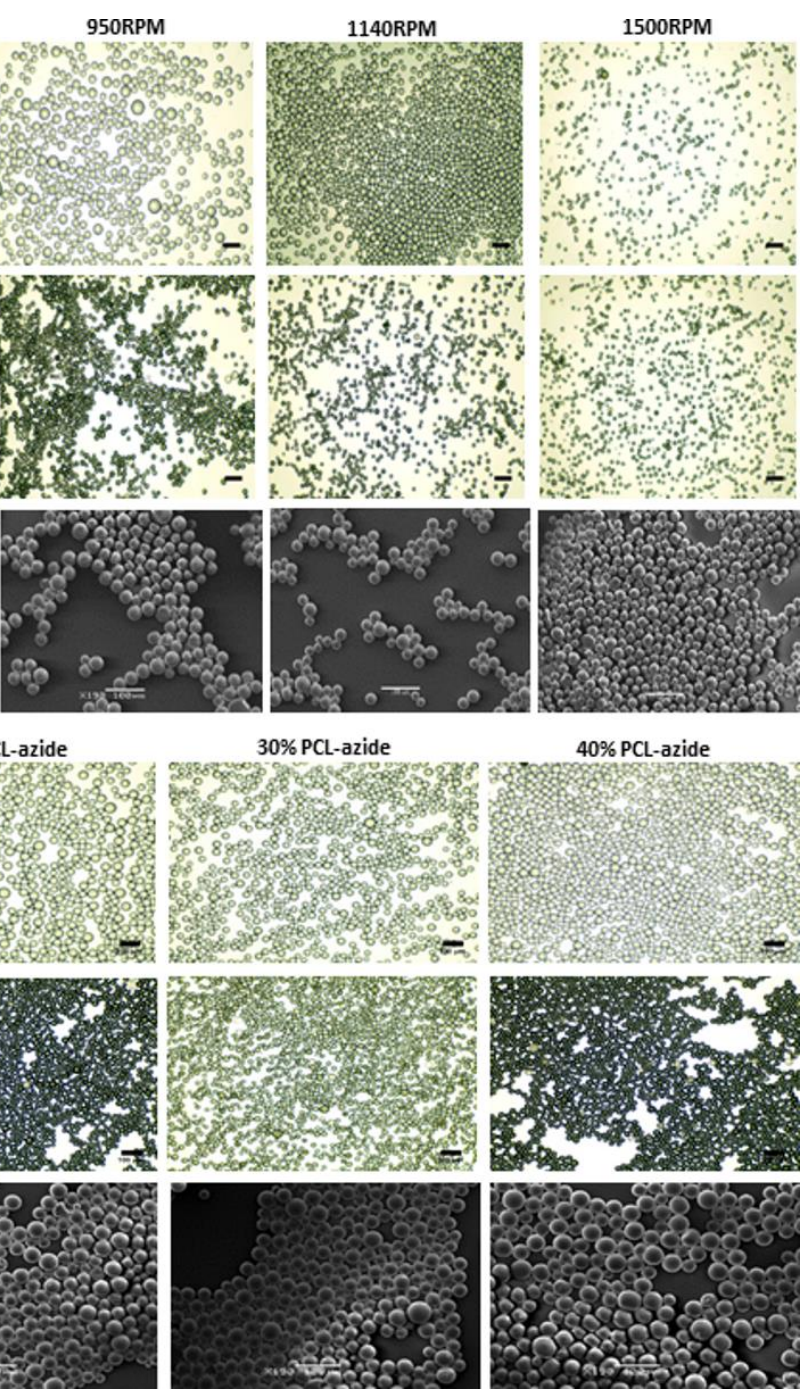

Figure 2: PCL-azide dissolved in DCM was injected into 1\%w/v PVA solution via syringe injection at a controlled rate. Particles were produced using either increasing stir speeds (Rows A-C) or increasing polymer concentrations (Rows D-F). Images show fresh particles (Rows A and D), solidified particles (Rows B and E) and freeze-dried particles visualised using Scanning electron microscopy (Rows C and F). All optical microscopy images taken at 10x objective. SEM shown at x190 magnification. All scale bars represent $100 \mu \mathrm{m}$.

At all PCL-azide concentrations the size distribution of particles was low at less than 0.5 indicating a highly monodisperse with a uniform, spherical morphology could be produced using membrane emulsification. Precise control over the resulting 
particle size was possible. The resulting particles were assessed for the presence of azide using IR and were all found to contain the indicative azide peak at $2100 \mathrm{~cm}^{-1}$, showing no degradation during the production process (data not shown).

To demonstrate the application of PCL-azide particles for the delivery of bioactive factors, we functionalised the model protein Human Serum Albumin (HSA) with a click chemistry unit, containing an internal alkyne, which can readily react with the azide functionality of PCL particles (Figure 1, D). With one-step modification in mind, we opted to use a copper-free click chemistry reagent, which is commercially available. Dibenzylcyclooctyne-peg4-maleimide (DBCO-mal) is readily available to react with the free thiol present in the cysteine at residue 34 of HSA (Figure 1, C). ${ }^{20}$ The resulting conjugate was analysed by HPLC (see supporting information, S3) and liquid chromatography mass spectrometry (LC-MS) (see supporting information, S4). The native unconjugated protein was identified by a sharp peak at $66559 \mathrm{Da}$ MW by LC-MS as expected. ${ }^{21}$ The conjugation reaction was successful with $73 \%$ relative abundance of the protein labelled, as shown by the additional peaks increasing in $\mathrm{MW}$ by $680 \mathrm{Da}$ corresponding to the addition of DBCO-mal (see supporting information, S4).

By labelling HSA with a fluorescent tag, we were successfully able to monitor the speed and efficiency of the click reaction of DBCO functionalised protein onto the surface of PCL-azide microparticles. HSA was tagged with fluorescent isothiocyanate

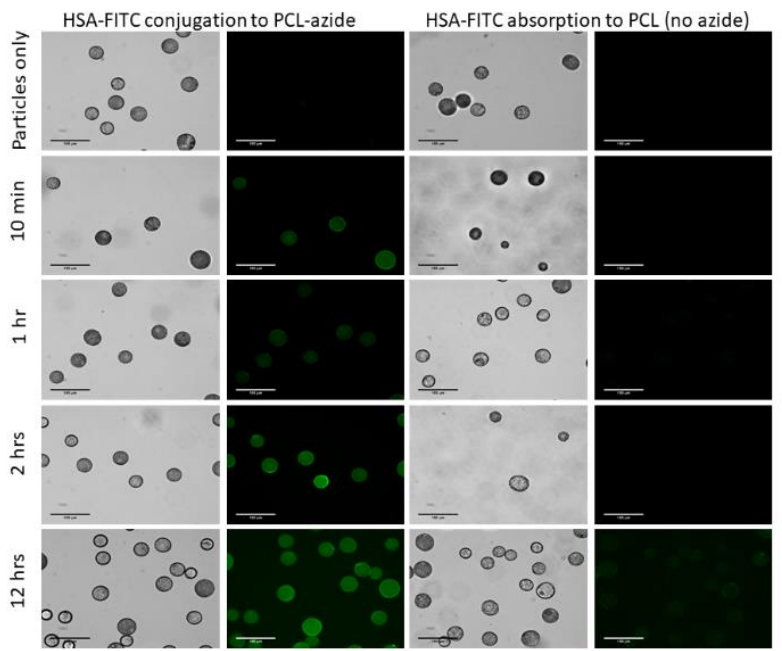

Figure 3: PCL-azide and PCL microparticles reacted with FITC labelled Human Serum Albumin at 1:1 molar ratio for increasing amounts of time. PCL-azide contains azide functional groups, which can react with the internal alkyne present on the DBCO linker tethered to the Human Serum Albumin via free cysteine. This copper-free click chemistry reaction occurs quickly and microparticles are fluorescent after just 10 minutes. Commercial PCL lacks the azide functional group, but fluorescence occurs if the reaction is carried out for a sufficient length of time to allow physical adsorption. All Images taken at $\times 20$ magnification. All scale bars represent $100 \mu \mathrm{m}$.

and DBCO-mal to produce a fluorescently tagged protein that could be used in the click reaction. All unbound reactants were removed using a PD10 desalting column and the fluorescent conjugates were mixed with PCL-azide Microparticles at a ratio of 1:1 mole equivalents (in respect to the polymer) for 10 minutes, 1 hour, 2 hour, and 12 hour time points. After reaction, the particles were washed with distilled water and fluorescent images were taken (Figure 3). It was found that the immobilisation of protein onto the surface of the PCL-azide microparticles occurred within 10 minutes compared to 12 hours for non-functionalised PCL controls. Microparticles displayed fluorescence and therefore the presence of HSA protein at all time points (Figure 3). Azide-deficient PCL, which lacks the reactive groups needed for the attachment of the DBCO linker, was used as a control. Fluorescence was only seen on these particles after 12 hours; by this point physical absorption of water onto the surface of the particles could be responsible for the fluorescence seen, as opposed to a conjugation reaction occurring.

To summarise, here we describe a readily adaptable template for the immobilisation of biological factors that can be applied to a large variety of widely available polyester polymers. We have demonstrated the facile production of surface-clickable microcarriers with the simple synthesis of commercially available polymers, functionalised with useful terminal groups. We have demonstrated the one step copper-free click chemistry reaction can be carried out with high efficiency in 10 minutes in physiological conditions. It can be used for the immobilisation of proteins and other bioactive factors to the surface of a microcarrier delivery system. The DBCO reagent is commercially available with a variety of reactive functional groups that can be used to conjugate to amines, carbonyls, and thiols. This means that the techniques discussed here can easily be adapted and adjusted to allow conjugation to a whole range of compounds. The combination of surface active microcarriers with efficient and specific DBCO reagents offers a new way of loading and delivering bioactive agents, with potential applications in both drug delivery and tissue engineering.

\section{- CONFLICTS OF INTEREST}

There are no conflicts to declare

\section{- ACKNOWLEDGEMENTS}

The authors are grateful for the funding provided by the EPSRC ICASE award (grant EP/L505729/I).

\section{- SUPPORTING INFORMATION}

Detailed experimental procedures and additional figures; synthesis of the PCL-Azide polymer; Strain promoted AzideAlkyne Cycloaddition; Microparticle production; synthesis of DBCO-peg4-maleimide-HSA conjugates; synthesis of HSA immobilized PCL-N 3 microparticles; HPLC analysis of HSA and DBCO-peg4-maleimide; LCMS analysis of the HSA conjugation with DBCO-peg4-maleimide; DOSY-NMR spectra of DBCO-acid and corresponding reaction of DBCO-acid with PCL-N $\mathrm{N}_{3}$ and PCL.

\section{- AUTHOR INFORMATION}

Corresponding author

*Aram.Saeed@uea.ac.uk 
- Notes

The Authors declare no competing financial interest

\section{- REFERENCES}

(1) de Luca, A. C., Terenghi, G., and Downes, S. (2014) Chemical surface modification of poly-epsilon-caprolactone improves Schwann cell proliferation for peripheral nerve repair. Journal of tissue engineering and regenerative medicine 8, 153-63.

(2) Rice, J. J., Martino, M. M., De Laporte, L., Tortelli, F., Briquez, P. S., and Hubbell, J. A. (2013) Engineering the regenerative microenvironment with biomaterials. Advanced healthcare materials 2, 57-71.

(3) Walden, G., Liao, X., Donell, S., Raxworthy, M. J., Riley, G. P., and Saeed, A. (2017) A Clinical, Biological, and Biomaterials Perspective into Tendon Injuries and Regeneration. Tissue engineering. Part B, Reviews 23, 44-58.

(4) Lim, S. I., Hahn, Y. S., and Kwon, I. (2015) Site-specific albumination of a therapeutic protein with multi-subunit to prolong activity in vivo. Journal of controlled release : official journal of the Controlled Release Society 207, 93-100.

(5) Kirby, G. T. S., White, L. J., Steck, R., Berner, A., Bogoevski, K., Qutachi, O., Jones, B., Saifzadeh, S., Hutmacher, D. W., Shakesheff, K. M., and Woodruff, M. A. (2016) Microparticles for Sustained Growth Factor Delivery in the Regeneration of Critically-Sized Segmental Tibial Bone Defects. Materials (Basel, Switzerland) 9.

(6) Moshiri A, O. A. ((2013) Tendon and Ligament Tissue Engineering, Healing and Regenerative Medicine. Sports Med Doping Stu 3:126.

(7) Saeed, A. O., Magnusson, J. P., Moradi, E., Soliman, M., Wang, W., Stolnik, S., Thurecht, K. J., Howdle, S. M., and Alexander, C. (2011) Modular construction of multifunctional bioresponsive cell-targeted nanoparticles for gene delivery. Bioconjug Chem $22,156-68$

(8) Mondschein, R. J., Kanitkar, A., Williams, C. B., Verbridge, S. S., and Long, T. E. (2017) Polymer structure-property requirements for stereolithographic $3 \mathrm{D}$ printing of soft tissue engineering scaffolds. Biomaterials 140, 170-188.

(9) B. Dahndayuthapani, Y. Y., Toru M, and D. Sakthi Kumar (2011) Polymeric Scaffolds in Tissue Engineering Application: A Review. International Journal of Polymer Science 2011, 19 pages.

(10) Place, E. S., George, J. H., Williams, C. K., and Stevens, M. M. (2009) Synthetic polymer scaffolds for tissue engineering. Chemical Society reviews 38, 1139-51.

(11) Saeed, A. O., Dey, S., Howdle, S. M., Thurecht, K. J., and Alexander, C. (2009) One-pot controlled synthesis of biodegradable and biocompatible co-polymer micelles. Journal of Materials Chemistry 19, 4529-4535

(12) Lenoir, S., Riva, R., Lou, X., Detrembleur, C., Jérôme, R., and Lecomte, P. (2004) Ring-Opening Polymerization of $\alpha$-Chloro$\varepsilon$-caprolactone and Chemical Modification of Poly $(\alpha$-chloro- $\varepsilon$ caprolactone) by Atom Transfer Radical Processes. Macromolecules 37, 4055-4061.
(13) Shi, Y., Schmalz, H., and Agarwal, S. (2015) Designed enzymatically degradable amphiphilic conetworks by radical ring-opening polymerization. Polymer Chemistry 6, 6409-6415.

(14) Lou, X., Detrembleur, C., Lecomte, P., and Jérôme, R. (2001) Living Ring-Opening (Co)polymerization of 6,7-Dihydro$2(5 \mathrm{H})$-oxepinone into Unsaturated Aliphatic Polyesters. Macromolecules 34, 5806-5811.

(15) Zhu, Y., Gao, C., Liu, X., and Shen, J. (2002) Surface modification of polycaprolactone membrane via aminolysis and biomacromolecule immobilization for promoting cytocompatibility of human endothelial cells. Biomacromolecules 3, 1312-9.

(16) Bell, C. A., Hedir, G. G., O'Reilly, R. K., and Dove, A. P. (2015) Controlling the synthesis of degradable vinyl polymers by xanthate-mediated polymerization. Polymer Chemistry 6, 74477454.

(17) Urbaniak, T., and Musial, W. (2016) Methods for obtaiing polyepsilon-caprolactone for producing micro-and nanoscale drug carriers. Acta poloniae pharmaceutica 73, 811-825.

(18) Cambronero-Rojas, A., Torres-Vergara, P., Godoy, R., von Plessing, C., Sepúlveda, J., and Gómez-Gaete, C. (2015) Capreomycin oleate microparticles for intramuscular administration: Preparation, in vitro release and preliminary in vivo evaluation. Journal of controlled release : official journal of the Controlled Release Society 209, 229-237

(19) Dragosavac, M. M., Holdich, R. G., Vladisavljević, G. T., and Sovilj, M. N. (2012) Stirred cell membrane emulsification for multiple emulsions containing unrefined pumpkin seed oil with uniform droplet size. Journal of Membrane Science 392-393, 122-129.

(20) Simon, M., Zangemeister-Wittke, U., and Plückthun, A. (2012) Facile Double-Functionalization of Designed Ankyrin Repeat Proteins using Click and Thiol Chemistries. Bioconjugate Chemistry 23, 279-286.

(21) Anguizola, J., Matsuda, R., Barnaby, O. S., Hoy, K. S., Wa, C., DeBolt, E., Koke, M., and Hage, D. S. (2013) Review: Glycation of human serum albumin. Clinica chimica acta; international journal of clinical chemistry 425, 64-76. 